\title{
K-THEORETIC GROMOV-WITTEN INVARIANTS IN GENUS 0 AND INTEGRABLE HIERARCHIES
}

\author{
TODOR MILANOV AND VALENTIN TONITA
}

\begin{abstract}
We prove that the genus-0 invariants in K-theoretic GromovWitten theory are governed by an integrable hierarchy of hydrodynamic type. If the K-theoretic quantum product is semisimple, then we also prove the completeness of the hierarchy.
\end{abstract}

\section{Contents}

1. Introduction

1.1. Frobenius-like structure and the $S$-matrix

1.2. Commuting flows

1.3. Integrable hierarchies

1.4. The topological solution

1.5. Example: $X=p t$

1.6. Acknowledgements

2. Proof of Theorem 1.1

2.1. The derivations are Hamiltonian

2.2. The Hamiltonians are in involution

2.3. Canonical coordinates

2.4. Completeness

3. Construction of the topological solution

3.1. From descendants to ancestors 13

3.2. Genus-0 reconstruction in terms of the $S$-matrix 14

3.3. Proof of Theorem $1.3 \quad 15$

3.4. The Topological recursion relations 16

3.5. Geometric proof of TRR 1

References 18

\section{INTRODUCTION}

Let $X$ be a smooth complex projective variety and $K(X)=K^{0}(X ; \mathbb{C})$ be the Grothendieck group of topological vector bundles on $X$. Let us denote by $X_{0, n, d}$ the moduli stack of genus- 0 stable maps of degree $d \in H_{2}(X ; \mathbb{Z})$ with $n$ marked points. The operation that assigns to a point in the moduli space the cotangent 
line at the $i$-th marked point is functorial and it gives rise to a line bundle $L_{i}$, while evaluation at the $i$-th marked point gives rise to a map of Deligne-Mumford stacks ev $i_{i}: X_{0, n, d} \rightarrow X$ known as the evaluation map. If $E_{1}, \ldots, E_{n} \in K(X)$, then following Givental and Y. P. Lee (see [4, 9]) we introduce the K-theoretic Gromov-Witten invariant

$$
\left\langle E_{1} L_{1}^{k_{1}}, \ldots, E_{n} L_{n}^{k_{n}}\right\rangle_{g, n, d}=\chi\left(\mathcal{O}_{\mathrm{virt}} \otimes \mathrm{ev}_{1}^{*}\left(E_{1}\right) L_{1}^{k_{1}} \cdots \mathrm{ev}_{n}^{*}\left(E_{n}\right) L_{n}^{k_{n}}\right),
$$

where $\chi(\mathcal{F})$ denotes the holomorphic Euler characteristic of $\mathcal{F}$ and $\mathcal{O}_{\text {virt }}$ is the so called virtual structure sheaf (see [9]).

Let us fix a set $P_{1}, \ldots, P_{r}$ of ample line bundles, s.t., $p_{i}=c_{1}\left(P_{i}\right)$ form a basis of $H_{2}(X ; \mathbb{Z}) /$ torsion. If $d \in H_{2}(X ; \mathbb{Z})$ then we define

$$
Q^{d}:=Q_{1}^{\left\langle p_{1}, d\right\rangle} \cdots Q_{r}^{\left\langle p_{r}, d\right\rangle}
$$

where $Q_{1}, \ldots, Q_{r}$ are formal variables known as the Novikov variables. For $t \in$ $K(X)$ put

$$
\left\langle E_{1} L_{1}^{k_{1}}, \ldots, E_{n} L_{n}^{k_{n}}\right\rangle_{g, n}(t)=\sum_{d} \sum_{\ell=0}^{\infty} \frac{Q^{d}}{\ell !}\left\langle E_{1} L_{1}^{k_{1}}, \ldots, E_{n} L_{n}^{k_{n}}, t, \ldots, t\right\rangle_{g, n+\ell, d} .
$$

1.1. Frobenius-like structure and the $S$-matrix. Let us fix a basis $\left\{\Phi_{i}\right\}_{i=0}^{N} \subset$ $K(X)$ and write $t=\sum_{i=0}^{N} t_{i} \Phi_{i}$. In general, the RHS of (1) is a formal power series in $Q_{i}(1 \leq i \leq r)$ and $t_{j}(0 \leq j \leq N)$. We are going to assume that there exists an open subset

$$
B=\left\{(Q, t)|| Q_{i}|<\epsilon,| t_{j} \mid<\epsilon\right\} \subset \mathbb{C}^{r} \times \mathbb{C}^{N+1},
$$

in which the series (1) is convergent for all possible choices of the insertions $E_{i} L_{i}^{k_{i}}$. This condition is satisfied in many important cases, such as when $X$ is a projective space or a flag manifold (see [8]). Our results are true in general as well, but we have to replace $B$ with the formal germ of $\mathbb{C}^{r} \times \mathbb{C}^{N+1}$ at $(0,0)$. Slightly abusing the notation we denote by $T B$ the relative tangent bundle of the projection $B \rightarrow \mathbb{C}^{r}$, $(Q, t) \mapsto Q$.

The vector space $K(X)$ is equipped with the Euler pairing

$$
g\left(\Phi_{i} \otimes \Phi_{j}\right):=g_{i j}:=\int_{X} \operatorname{ch}\left(\Phi_{i}\right) \operatorname{ch}\left(\Phi_{j}\right) \operatorname{Td}(X) .
$$

Put

$$
G\left(\Phi_{i} \otimes \Phi_{j}\right):=G_{i j}(t):=g_{i j}+\left\langle\Phi_{i}, \Phi_{j}\right\rangle_{0,2}(t) .
$$

The $K$-theoretic quantum product is defined by

$$
G\left(\Phi_{i} \bullet \Phi_{j}, \Phi_{k}\right)=\left\langle\Phi_{i}, \Phi_{j}, \Phi_{k}\right\rangle_{0,3}(t) .
$$

Using the natural trivialization $T B=B \times K(X), \partial / \partial t_{i} \mapsto \Phi_{i}$, we can identify $g$ and $G$ with non-degenerate bilinear forms on $T B$ and $\bullet$ with a multiplication on $T B$. It is known that the multiplication • and the pairing $G$ satisfy Dubrovin's 
axioms of a Frobenius manifold (see [1]) except for flat identity and Euler vector field (see [4]).

Following Givental [4], we introduce the operator series $S \in \operatorname{End}(K(X)) \otimes$ $\mathbb{C}(q) \llbracket Q, t \rrbracket$ defined by

$$
G\left(\Phi_{i}, S(t, q) \Phi_{j}\right)=S_{i j}\left(t, q^{-1}\right)
$$

where

$$
S_{i j}(t, q):=g_{i j}+\left\langle\Phi_{i}, \frac{\Phi_{j}}{1-q L}\right\rangle_{0,2}(t) .
$$

Here $q$ is a complex number such that $(1-q L)$ is invertible in $K\left(X_{0, n, d}\right)$ for all $n$ and $d$. It is known that the coefficient in front of a fixed monomial in $Q$ and $t$ of $S_{i j}(t, q)$ is a rational function in $q$ which vanishes at $q=\infty$ and has poles only at the roots of unity. The operator series $S(t, q)$ is a fundamental solution to the following system of differential equations

$$
(q-1) \partial_{t_{i}} S(t, q)=\Phi_{i} \bullet \bullet_{t} S(t, q), \quad 0 \leq i \leq N .
$$

In particular, the formulas

$$
\nabla_{\partial / \partial t_{i}}:=\partial_{t_{i}}-(q-1)^{-1} \Phi_{i} \bullet, \quad 0 \leq i \leq N,
$$

define a family of flat connections on $T B$. It is easy to check that for $q=-1$ the connection $\nabla$ turns into the Levi-Cevita connection for the pairing $G$, which in particular proves that the bilinear pairing $G$ is flat.

Motivated by Dubrovin's construction of the principal hierarchies in the theory of Frobenius manifolds (see 1, 3]), we introduce a hierarchy of differential equations on the formal loop space $\mathcal{L}(B)$. It is convenient however to work first purely algebraically using the language of differential algebras and then reformulate the construction using the geometry of the formal loop space.

1.2. Commuting flows. Let $\mathbb{C}\left[Q, v, \partial v, \partial^{2} v, \ldots\right]$ be the free differential algebra over the ring $\mathbb{C}[Q]$ on the vector variable $v=\left(v_{0}, \ldots, v_{N}\right)$. We refer to $\partial^{k} v=$ $\left(\partial^{k} v_{0}, \ldots, \partial^{k} v_{N}\right)$ with $k>0$ as jet variables. Each $\partial^{k} v_{i}$ should be viewed as a formal variable, $\partial^{0} v_{i}:=v_{i}$, and the differentiation is defined in the obvious way $\partial\left(\partial^{k} v_{i}\right)=\partial^{k+1} v_{i}$. Let us denote by $\mathcal{A}$ the completion of the differential algebra consisting of polynomials in the jet variables whose coefficients are convergent power series for $(Q, v) \in B$. If $q \in \mathbb{C} \backslash\{0\}$ is not a root of unity, then we define derivations $T_{i}(q)(0 \leq i \leq N)$ of $\mathcal{A}$, such that $T_{i}(q)$ commutes with $\partial$ and

$$
T_{i}(q) v:=\partial\left(S(v, q) \Phi_{i}\right)=(q-1)^{-1} \partial v \bullet S(v, q) \Phi_{i},
$$

where both sides take value in $\mathcal{A} \otimes K(X)$ via the identification $v:=\sum_{i=0}^{N} v_{i} \Phi_{i}$, and the derivation $\partial$ of $\mathcal{A}$ is extended uniquely to a derivation on $\mathcal{A} \otimes K(X)$, such that $\partial\left(1 \otimes \Phi_{i}\right)=0$. 
Following Dubrovin and Novikov (see [2]) we equip the vector space $\mathcal{F}:=\mathcal{A} / \partial \mathcal{A}$ with a Poisson bracket of hydrodynamic type

$$
\left\{f_{1}, f_{2}\right\}=\sum_{i, j=0}^{N} \int \frac{\delta f_{1}}{\delta v_{i}} A^{i j} \frac{\delta f_{2}}{\delta v_{j}}, \quad f_{1}, f_{2} \in \mathcal{F},
$$

where $\int: \mathcal{A} \rightarrow \mathcal{F}$ is the projection map, $A^{i j}$ is the first order differential operator

$$
A^{i j}=G^{i j}(v) \partial-\sum_{s, k=0}^{N} G^{i s} \Gamma_{s k}^{j}(v) \partial v_{k},
$$

$G^{i j}$ are the entries of the inverse of the matrix $\left(G_{i j}\right)$,

$$
\Gamma_{s k}^{j}(v)=\frac{1}{2} \sum_{\ell=0}^{N} G^{j \ell} G\left(\Phi_{s} \bullet \Phi_{k}, \Phi_{\ell}\right)
$$

are the Christoffel's symbols of the pairing $G$, and

$$
\frac{\delta}{\delta v_{i}}=\sum_{\ell=0}^{\infty}(-\partial)^{\ell} \frac{\partial}{\partial\left(\partial^{\ell} v_{i}\right)}
$$

is the variational derivative. Recall that $\delta / \delta v_{i} \circ \partial=0$ (in $\mathcal{A}$ ), so the variational derivative descends to the quotient $\mathcal{F}$.

Given $H \in \mathcal{F}$, we can define a derivation $X_{H}$ of $\mathcal{A}$, such that it commutes with $\partial$ and

$$
X_{H}\left(v_{i}\right)=\sum_{j=0}^{N}\left(G^{i j}(v) \partial-\sum_{s, k=0}^{N} G^{i s}(v) \Gamma_{s k}^{j}(v) \partial v_{k}\right) \frac{\delta H}{\delta v_{j}} .
$$

The map $H \rightarrow X_{H}$ turns $\mathcal{A}$ into a Poisson $\mathcal{F}$-module:

$$
\left[X_{H_{1}}, X_{H_{2}}\right]=-X_{\left\{H_{1}, H_{2}\right\}}, \quad H_{1}, H_{2} \in \mathcal{F} .
$$

We will refer to $H$ as Hamiltonian and to $X_{H}$ as Hamiltonian derivation. We say that the Hamiltonian derivation $X_{H}$ is polynomial in $v_{0}$ if the differential polynomial $X_{H}(v)$ depends polynomially in $v_{0}$. Our first result can be stated as follows.

Theorem 1.1. a) The derivations $T_{i}(q)(i=0,1, \ldots, N)$ are Hamiltonian and $T_{i}\left(q_{1}\right)$ and $T_{j}\left(q_{2}\right)$ commute for all $i, j, q_{1}$, and $q_{2}$.

b) If the K-theoretic quantum product is semisimple, then every Hamiltonian derivation $X_{H}$ commuting with $T_{i}(q)$ for all $i$ and polynomial in $v_{0}$ has the form

$$
X_{H}(v)=-\operatorname{Res}_{q=\infty} \partial S(v, q) C(q) d q, \quad C(q) \in K(X)[q] .
$$

Let us point out that the Hamiltonian corresponding to the derivation $T_{i}(q)$ is

$$
H_{i}(v, q):=\frac{2}{1+q}\left(g\left(v, \Phi_{i}\right)+\left\langle\frac{\Phi_{i}}{1-q^{-1} L}\right\rangle_{0,1}(v)\right) \quad(\bmod \partial \mathcal{A}) .
$$


Part a) follows immediately from the fact that $S(v, q)$ is a symplectic transformation solving the K-theoretic quantum differential equations, while for part b) we follow the argument of the proof of Lemma 3.6.7 and Lemma 3.6.20 in [3].

1.3. Integrable hierarchies. Theorem 1.1 allows us to construct an integrable hierarchy of evolutionary PDE on the loop space $\mathcal{L}(B):=C^{\infty}\left(S^{1}, B\right)$. Let us parametrize $S^{1}$ via $x \mapsto e^{2 \pi \sqrt{-1} x}$. Recall that a local functional is a function on $\mathcal{L}(B)$ of the form

$$
v(x) \mapsto \int_{0}^{1} P\left(x, v(x), \partial_{x} v(x), \ldots\right) d x,
$$

where

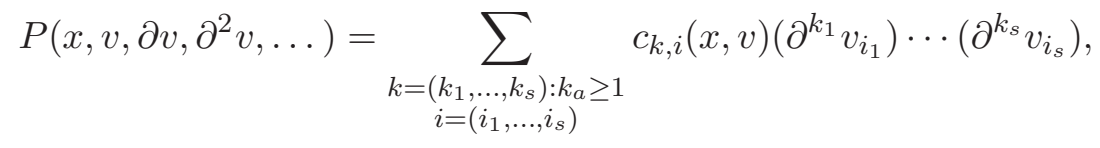

is a polynomial in the jet variables whose coefficients $c_{k, i}(x, v) \in C^{\infty}\left(S^{1} \times B\right)$. In particular, the elements of $\mathcal{F}$ are local functionals and the definition of the Poisson bracket of hydrodynamic type extends to the entire space of local functionals. The loop space $\mathcal{L}(B)$ has also another class of functions that comes from the evaluation map

$$
\text { ev }: S^{1} \times \mathcal{A} \rightarrow C^{\infty}(\mathcal{L}(B))
$$

defined by

$$
\mathrm{ev}(x, P)(v)=P\left(v(x), \partial_{x} v(x), \partial_{x}^{2} v(x), \ldots\right) .
$$

Following the notation in physics we write the evaluation maps as

$$
\mathrm{ev}(x, P)(v)=\int_{0}^{1} P\left(v(y), \partial_{y} v(y), \partial_{y}^{2} v(y), \ldots\right) \delta(x-y) d y,
$$

which allows us to think of $\mathrm{ev}(x, P)$ as a local functional and to extend the definition of the Poisson bracket. Note that we have

$$
\{\mathrm{ev}(x, P), H\}=\mathrm{ev}\left(x, X_{H}(P)\right), \quad P \in \mathcal{A}, \quad H \in \mathcal{F},
$$

which justifies why the derivation $X_{H}$ was called Hamiltonian. Put

$$
P_{n, i}(v, \partial v):=-\operatorname{Res}_{q=\infty} d q(q-1)^{n} T_{i}(q) v, \quad 0 \leq i \leq N, \quad n \geq 0 .
$$

Let us introduce the following system of ODEs

$$
\partial_{t_{n, i}} v=P_{n, i}\left(v, \partial_{x} v\right), \quad 0 \leq i \leq N, \quad n \geq 0,
$$

where

$$
v=v(x, \mathbf{t}), \quad \mathbf{t}=\left(t_{n, i}\right)_{n=0,1,2, \cdots}^{i=0, \ldots, N} .
$$

Note that the first equation of the hierarchy is $\partial_{0,0} v=\partial_{x} v$. Therefore, we may assume that $x=t_{0,0}$. 
Corollary 1.2. The equations (3) are Hamiltonian and pairwise compatible. If the quantum K-product is semisimple, then (3) is a completely integrable system.

The Hamiltonians corresponding to (3) are given by

$$
H_{n, i}(v):=-\operatorname{Res}_{q=\infty} d q(q-1)^{n} H_{i}(v, q) .
$$

The Taylor's series expansion of $S(v, q)$ near $q=\infty$ has the form

$$
S(v, q)=1+\sum_{n=1}^{\infty} S_{n}(v)(q-1)^{-n} .
$$

The quantum differential equations imply that $\partial_{v_{0}} S_{n}(v)=S_{n+1}(v)$. From this relation we get that $S_{n}(v)$ depends polynomially on $v_{0}$. By definition

$$
X_{H_{n, i}}(v)=P_{n, i}(v, \partial v)=-\operatorname{Res}_{q=\infty} d q(q-1)^{n-1} \partial v \bullet S(v, q) \Phi_{i}=\partial v \bullet S_{n}(v) \Phi_{i},
$$

so the Hamiltonian derivations $X_{H_{n, i}}(v)$ are polynomial in $v_{0}$. Corollary 1.2 is a direct consequence of Theorem 1.1.

1.4. The topological solution. The second goal of our paper is to construct a solution to the system (3) in terms of genus-0 K-theoretic GW invariants. The genus-0 total descendant potential is defined by

$$
\mathcal{F}(\mathbf{t})=\sum_{n=0}^{\infty} \frac{1}{n !}\left\langle\mathbf{t}\left(L_{1}\right), \ldots, \mathbf{t}\left(L_{n}\right)\right\rangle_{0, n},
$$

where $\mathbf{t}(q) \in K(X)\left[q, q^{-1}\right]$. Let us define

$$
\mathbf{t}(q):=\sum_{k=0}^{\infty} \sum_{i=0}^{N} t_{k, i} \Phi_{i}(q-1)^{k}
$$

where $t_{k, i}$ are formal variables. Let us denote $\partial_{m, i}:=\partial / \partial t_{m, i}$. Recall the Ktheoretic J-function of $X$

$$
J(v, q)=1-q+v+\sum_{i=0}^{N} \Phi^{i}\left\langle\frac{\Phi_{i}}{1-q L}\right\rangle_{0,1}(v)=(1-q) S(v, q)^{-1} \mathbf{1},
$$

where $v \in K(X)$ and $\Phi^{i}:=\sum_{j=0}^{N} g^{i j} \Phi_{j}(0 \leq i \leq N)$ is the basis of $K(X)$ dual to $\left\{\Phi_{i}\right\}_{i=0}^{N}$ with respect to the Euler pairing $g$. Using the $J$-function we introduce new local coordinates $w=\sum_{a=0}^{N} w_{a} \Phi^{a}$ on $B$

$$
w=J(v, 0)-1=v+\sum_{i=0}^{N}\left\langle\Phi_{i}\right\rangle_{0,1}(v) \Phi^{i}
$$

Note that the vector fields $\partial / \partial w_{a}=\sum_{b} G^{a b}(v) \partial / \partial v_{b}$ correspond to the differential forms $d v_{a}$ under the identification $T B \cong T^{*} B$ defined via the bilinear pairing $G$. 
Theorem 1.3. The functions

$$
w_{a}(\mathbf{t})=\partial_{0, a} \partial_{0,0} \mathcal{F}(\mathbf{t}), \quad 0 \leq a \leq N,
$$

provide a solution to the integrable hierarchy obtained from (3) via the Miura transformation (4).

1.5. Example: $X=p t$. In this case, the quantum differential equations are straightforward to solve. We have

$$
S(v, q)=e^{v /(q-1)}, \quad J(v, q)=e^{v /(1-q)} .
$$

The integrable system (3) takes the form

$$
\partial_{n} v=\frac{v^{n}}{n !} \partial v, \quad n=0,1, \ldots,
$$

i.e., this is the dispersionless KdV hierarchy. The Miura transformation is $w=$ $e^{v}-1$, so the dispersionless $\mathrm{KdV}$ hierarchy is transformed into

$$
\partial_{n} w=\frac{1}{n !}(\log (1+w))^{n} \partial w, \quad n=0,1, \ldots
$$

The topological solution is

$$
w(\mathbf{t})=\sum_{n=1}^{\infty} \frac{1}{n !}\langle 1,1, \mathbf{t}(L), \ldots, \mathbf{t}(L)\rangle_{0, n+2},
$$

where

$$
\mathbf{t}(L)=\sum_{n=0}^{\infty} t_{n}(L-1)^{n} .
$$

Using the above equation and the string equation we can compute all genus 0 K-theoretic GW invariants of the point. Our answer agrees with the answer of Y.P. Lee [10]. It is very tempting to conjecture that the higher genus deformation of the topological solution

$$
\widehat{w}(\hbar, \mathbf{t}):=\sum_{g, n=0}^{\infty} \frac{\hbar^{g}}{n !}\langle 1,1, \mathbf{t}(L), \ldots, \mathbf{t}(L)\rangle_{g, n+2},
$$

is a solution to an integrable hierarchy which is a Miura transform of the $\mathrm{KdV}$ hierarchy.

While the higher-genus K-theoretic GW invariants of the point are still very difficult to compute, motivated by the above example, we would like to investigate further the relation of the hierarchy (3) with the genus- 0 hierarchies in cohomological GW theory. Especially, the adelic characterization of genus 0 K-theoretic GW invariants (see [5]) seems to be appropriate to study such a question. In particular, it would be interesting to find out if the integrable hierarchy (3) is bi-Hamiltonian. We are planning to investigate these questions in a future publication. 
1.6. Acknowledgements. We are thankful to Y. Zhang for showing interest in our work and for several useful discussions. The work of T. M. is partially supported by JSPS Grant-In-Aid 26800003 and by the World Premier International Research Center Initiative (WPI Initiative), MEXT, Japan. This work started during a visit of the second author to Kavli-IPMU. V. T. would like to thank the institute for hospitality and support.

\section{Proof of Theorem 1.1}

The proof of part a) will be given in Sections 2.1 and 2.2. The argument for part b) will be given in Section 2.4.

2.1. The derivations are Hamiltonian. The fact that the derivation $T_{i}(q)$ is Hamiltonian with Hamiltonian (2) follows from the following formula:

$$
\left\{f, H_{i}\right\}=\int \sum_{a, s=0}^{N} G\left(\partial S(v, q) \Phi_{i}, \Phi_{s}\right) G^{s a} \frac{\delta f}{\delta v_{a}} .
$$

Therefore, we just need to prove (5).

Let us denote by $g=\left(g_{a b}\right)$ and $G=\left(G_{a b}\right)$ the matrices of the two pairings. The pairing $G$ can be expressed in terms of $g$ and $S(v, 0)$. Indeed, we have the following identity expressing the fact that $S$ is a symplectomorphism (see [4, 8])

$$
g\left(a, S\left(v, q^{-1}\right)^{-1} b\right)=G(S(v, q) a, b), \quad a, b \in K(X) .
$$

If we set $q=0$, then since $S(v, \infty)=1$, we get

$$
g=G S(v, 0) \text {. }
$$

On the other hand,

$\frac{1}{2}(q+1) A^{a b} \frac{\delta H_{i}}{\delta v_{b}}=G^{a b} \partial G\left(S(v, q) \Phi_{i}, \Phi_{b}\right)-\sum_{s, \ell} G^{a s} \Gamma_{s \ell}^{b}(v) \partial v_{\ell} G\left(S(v, q) \Phi_{i}, \Phi_{b}\right)$.

Note that $\partial G^{-1}=-\sum_{\ell} \Omega_{\ell} G^{-1} \partial v_{\ell}$, where $\Omega_{\ell}=\Phi_{\ell} \bullet$ is the operator of quantum K-theoretic multiplication by $\Phi_{\ell}$. Therefore,

$$
G^{a b} \circ \partial=\partial \circ G^{a b}+\sum_{s, \ell}\left(\Omega_{\ell}\right)_{a s} G^{s b} \partial v_{\ell}
$$

By definition

$$
\sum_{k, \ell}\left(\Omega_{\ell}\right)_{a k} G^{k b} \partial v_{\ell}=2 \sum_{s, \ell} G^{a s} \Gamma_{s \ell}^{b}(v) \partial v_{\ell}
$$

So

$\frac{1}{2}(q+1) A^{a b} \frac{\delta H_{i}}{\delta v_{b}}=\partial\left(G^{a b} G\left(S(v, q) \Phi_{i}, \Phi_{b}\right)\right)+\frac{1}{2} \sum_{s, \ell}\left(\Omega_{\ell}\right)_{a s} G^{s b} \partial v_{\ell} G\left(S(v, q) \Phi_{i}, \Phi_{b}\right)$. 
K-THEORETIC GW INVARIANTS IN GENUS 0 AND INTEGRABLE HIERARCHIES

Note that

$\sum_{b}\left(\Omega_{\ell}\right)_{a s} G^{s b} G\left(S(v, q) \Phi_{i}, \Phi_{b}\right)=G\left(S(v, q) \Phi_{i}, \Phi_{\ell} \bullet \widetilde{\Phi}^{a}\right)=G\left(\Phi_{\ell} \bullet S(v, q) \Phi_{i}, \widetilde{\Phi}^{a}\right)$,

where $\widetilde{\Phi}^{a}=\sum_{b=0}^{N} G^{a b} \Phi_{b}$. Recalling the differential equation $(q-1) \partial_{v_{\ell}} S(v, q)=$ $\Phi_{\ell} \bullet S(v, q)$ we get

$$
\frac{1}{2}(q+1) \sum_{b} A^{a b} \frac{\delta H_{i}}{\delta v_{b}}=\frac{1}{2}(q+1) \sum_{b} G^{a b} G\left(\partial S(v, q) \Phi_{i}, \Phi_{b}\right)
$$

where we used that

$$
\sum_{b} \partial\left(G^{a b} G\left(x, \Phi_{b}\right)\right)=\sum_{b} \partial\left(g^{a b} g\left(x, \Phi_{b}\right)\right)=\sum_{b} G^{a b} G\left(\partial x, \Phi_{b}\right) .
$$

By definition $\left\{f, H_{i}\right\}=\int \sum_{a, b} \frac{\delta f}{\delta v_{a}} A^{a b} \frac{\delta H_{i}}{\delta v_{b}}$, so the above formula implies (5).

2.2. The Hamiltonians are in involution. We have to prove that

$$
\left\{H_{i}\left(v, q_{1}\right), H_{j}\left(v, q_{2}\right)\right\}=0, \quad \forall i, j=0, \ldots, N .
$$

Recalling formula (5) we get that the vanishing of the above bracket is equivalent to the fact that the differential polynomial

$$
G\left(\partial S(v, q) \Phi_{i}, S\left(v, q_{2}\right) \Phi_{j}\right)=\frac{1}{q_{1}-1} \sum_{\ell=0}^{N} g\left(S\left(v, q_{2}^{-1}\right)^{-1} \Omega_{\ell} S\left(v, q_{1}\right) \Phi_{i}, \Phi_{j}\right) \partial v_{\ell}
$$

is $\partial$-exact, where $\Omega_{\ell}$ is the linear operator of quantum multiplication by $\Phi_{\ell}$ and the equality is derived by using the quantum differential equations for $S\left(v, q_{1}\right)$ and the symplectic property of $S\left(v, q_{2}\right)$ (see (6) ). Recalling again the quantum differential equations for $S$ we get

$$
S\left(v, q_{2}^{-1}\right)^{-1} \Omega_{\ell} S\left(v, q_{1}\right)=\partial_{v_{\ell}}\left(\frac{\left(q_{1}-1\right)\left(q_{2}-1\right)}{q_{1} q_{2}-1}\left(S\left(v, q_{2}^{-1}\right)^{-1} S\left(v, q_{1}\right)-1\right)\right) .
$$

The above formula implies the $\partial$-exactness, because the pairing $g$ is constant.

2.3. Canonical coordinates. The notion of canonical coordinates of a semisimple Frobenius manifold (see [1]) is straightforward to generalize to the settings of quantum K-theory. In fact, the quantum K-ring is a F-manifold in the sense of Hertling and Manin (see [7]), so we can recall the notion of canonical coordinates of a F-manifold (see Corollary 5.2.1 in [6]). However, for the sake of completeness, let us work out the necessary details in our setting.

We say that the K-theoretic quantum product is semisimple at $v^{(0)} \in B$ if there are local holomorphic vector fields $e_{i}, 0 \leq i \leq N$, defined in an open neighborhood of $v^{(0)}$, such that

$$
e_{i} \bullet e_{j}=\delta_{i j} e_{j}, \quad i, j=0, \ldots N
$$


for all $v$ sufficiently close to $v^{(0)}$. Note that in the basis $\left\{e_{i}\right\}_{i=0}^{N}$ the pairing $G$ is diagonal

$$
G\left(e_{i}, e_{j}\right)=\Delta_{i}(v) \delta_{i j}
$$

where $\Delta_{i}(v) \neq 0$ for all $i$ and for all $v$ sufficiently close to $v^{(0)}$.

Lemma 2.1. If $v^{(0)} \in B$ is a semisimple point and $\left\{e_{i}\right\}_{i=0}^{N}$ is a corresponding basis of idempotents, then there are local coordinates $u_{i}, 0 \leq i \leq N$, such that $e_{i}=\partial / \partial u_{i}$.

Proof. We need to prove that the vector fields $e_{i}$ and $e_{j}$ commute. The quantum connection can be written in the form

$$
\nabla=\nabla^{\text {L.C. }}+\alpha \sum_{\ell=0}^{N} \Omega_{\ell}(v) d v_{\ell}, \quad \alpha:=-\frac{1}{2}-\frac{1}{q-1},
$$

where $\nabla^{\text {L.C. }}$ is the Levi-Civita connection for the bilinear pairing $G$. The flatness of $\nabla$ implies that

$$
\left(\nabla_{e_{i}} \nabla_{e_{j}}-\nabla_{e_{j}} \nabla_{e_{i}}\right) e_{k}=\nabla_{\left[e_{i}, e_{j}\right]} e_{k}
$$

Comparing the coefficients in front of $\alpha$ we get

$$
e_{i} \bullet\left(\nabla_{e_{j}}^{\text {L.C. }} e_{k}\right)+\nabla_{e_{i}}^{\text {L.C. }}\left(e_{j} \bullet e_{k}\right)-(i \leftrightarrow j)=\left[e_{i}, e_{j}\right] \bullet e_{k},
$$

where the expression in the brackets on the LHS is obtained from the preceeding expression by switching $i$ and $j$. Using that $e_{a}$ are idempotents we get

$$
\Gamma_{j k}^{i} e_{i}-\Gamma_{i k}^{j} e_{j}+\sum_{s=0}^{N}\left(\delta_{j k}-\delta_{i k}\right) \Gamma_{i j}^{s} e_{s}=\left[e_{i}, e_{j}\right] \bullet e_{k} .
$$

Multiplying both sides by $e_{k}$, we get that the LHS is 0 , while the RHS does not change, so $\left[e_{i}, e_{j}\right] \bullet e_{k}=0$ for all $k$ and we get $\left[e_{i}, e_{j}\right]=0$.

Let us denote by $\Psi$ the matrix with entries $\Psi_{a i}=\frac{\partial v_{a}}{\partial u_{i}}$, then the above lemma implies that

$$
\Psi^{-1} \Omega_{\ell} \Psi=\operatorname{Diag}\left(\frac{\partial u_{0}}{\partial v_{\ell}}, \ldots, \frac{\partial u_{N}}{\partial v_{\ell}}\right)
$$

2.4. Completeness. Let us assume that $f \in \mathcal{A}$ is such that $\left\{\int f, H_{i}(v, q)\right\}=0$ for all $i=0, \ldots, N$.

Lemma 2.2. The differential polynomial $f$ is independent of the jet variables, i.e., $\partial f / \partial\left(\partial^{k} v_{a}\right)=0$ for $k>0$.

Proof. Recalling formula (5) and using also that

$$
\sum_{b=0}^{N} G^{a b} G\left(x, \Phi_{b}\right)=\sum_{b=0}^{N} g^{a b} g\left(x, \Phi_{b}\right)
$$


we get that the differential polynomial

$$
\sum_{j=0}^{N} W^{j}(v, \partial v, \ldots) \partial g\left(S(v, q) \Phi_{i}, \Phi_{j}\right)
$$

is $\partial$-exact, where $W^{j}=\sum_{a} g^{j a} \frac{\delta f}{\delta v_{a}}$. Therefore the variational derivatives

$$
\frac{\delta}{\delta v_{a}}\left(\sum_{j=0}^{N} W^{j}(v, \partial v, \ldots) \partial g\left(S(v, q) \Phi_{i}, \Phi_{j}\right)\right)=0
$$

for all $a=0, \ldots, N$. By definition, the above variational derivative is the sum of

$$
\partial_{v_{a}}\left(\sum_{j} W^{j} \partial g\left(S(v, q) \Phi_{i}, \Phi_{j}\right)\right)-\partial \partial_{\partial v_{a}}\left(\sum_{j} W^{j} \partial g\left(S(v, q) \Phi_{i}, \Phi_{j}\right)\right)
$$

and

$$
\sum_{k \geq 2} \sum_{j=0}^{N}(-\partial)^{k}\left(\frac{\partial W^{j}}{\partial\left(\partial^{k} v_{a}\right)} \partial g\left(S(v, q) \Phi_{i}, \Phi_{j}\right)\right) .
$$

Let us denote by $\Omega_{\ell}$ the linear operator of quantum multipication by $\Phi_{\ell}$. Recalling also the quantum differential equation for $S$ and canceling a factor of $q-1$, we transform (77) and (86) respectively into

$$
\left.g\left(\sum_{j, \ell}\left(\partial_{v_{a}}\left(W^{j} \Omega_{\ell}\right) S-\partial\left(\partial_{\partial v_{a}} W^{j} \Omega_{\ell} S\right)\right) \Phi_{i}, \Phi_{j}\right) \partial v_{\ell}-\sum_{j=0}^{N} \partial\left(W^{j} \Omega_{a}\right) S \Phi_{i}, \Phi_{j}\right)
$$

and

$$
\left.\sum_{k \geq 2} \sum_{k^{\prime}=0}^{k}\left(\begin{array}{c}
k \\
k^{\prime}
\end{array}\right) \sum_{\ell=0}^{N} g\left((-\partial)^{k-k^{\prime}}\left(\frac{\partial W^{j}}{\partial\left(\partial^{k} v_{a}\right)} \Omega_{\ell} \partial v_{\ell}\right)(-\partial)^{k^{\prime}} S \Phi_{i}, \Phi_{j}\right)\right) .
$$

The sum of the above expressions can be written in the form $\sum_{j} g\left(A_{j} S \Phi_{i}, \Phi_{j}\right)=0$, where $A_{j}$ is a polynomial in $(q-1)^{-1}$ with coefficients in $\operatorname{End}(K(X))$. Since $g$ is a non-degenerate pairing, we can cancel $S$, i.e. we must have $\sum_{j=0}^{N} g\left(A_{j} \Phi_{i}, \Phi_{j}\right)=0$. Let $m$ be a maximal non-negative integer, s.t., $\partial_{\partial^{m} v_{a}} W^{j} \neq 0$ for some $j$. If $m \geq 2$, then the coefficient in front of $(q-1)^{-m}$ of $A_{j}$ is precisely

$$
(-1)^{m} \frac{\partial W^{j}}{\partial\left(\partial^{m} v_{a}\right)}\left(\sum_{\ell=0}^{N} \Omega_{\ell} \partial v_{\ell}\right)^{m+1} \text {. }
$$

If the quantum multiplication is semisimple, then we can find a matrix $\Psi=\Psi(v)$, holomorphically invertible for all $v$ in a neighborhood of a fixed semisimple point, such that

$$
\Psi^{-1} \Omega_{\ell} \Psi=\operatorname{Diag}\left(\frac{\partial u_{0}}{\partial v_{\ell}}, \ldots, \frac{\partial u_{N}}{\partial v_{\ell}}\right)
$$


where $u_{i}=u_{i}(v)(0 \leq i \leq N)$ are the corresponding canonical coordinates. Using again that $g$ is non-degenerate, we may replace $\Phi_{i}$ by $\Psi e_{i}$, where $e_{i}$ is the $i$ th standard vector. We get

$$
-\left(-\partial u_{i}\right)^{m+1} \sum_{j=0}^{N} \frac{\partial W^{j}}{\partial\left(\partial^{m} v_{a}\right)} g\left(\Psi e_{i}, \Phi_{j}\right)=0 .
$$

Let us choose a non-constant smooth loop $v: S^{1} \rightarrow B$, s.t., $v(x)$ is in a neighborhood of the fixed semisimple point for all $x \in S^{1}$ and

$$
\partial_{x} u_{i}(v(x)) \neq 0(0 \leq i \leq N), \quad \text { ev }\left(x, \frac{\partial W^{j}}{\partial\left(\partial^{m} v_{a}\right)}\right)(v) \neq 0
$$

for some $x \in S^{1}$ and some $j$. Such a choice is possible, because otherwise either $u_{i}(0 \leq i \leq N)$ will fail to be local coordinates or we will have a contradiction with the choice of $m$. However, evaluating (9) at $v(x)$ yields a contradiction with the non-degeneracy of $g$. Therefore, $m \leq 1$. A similar argument can be used to prove that $m$ can not be 1 .

Lemma 2.3. If $f(v) \in \mathcal{A}$ is independent of the jet variables, then vanishing of the brackets

$$
\left\{\int f(v), H_{i}(v, q)\right\}=0, \quad \forall i=0, \ldots, N
$$

is equivalent to the following system of equations

$$
\frac{\partial^{2} f}{\partial v_{i} \partial v_{a}}=\sum_{k=0}^{N}\left(\Omega_{i}\right)_{k a} \frac{\partial^{2} f}{\partial v_{k} \partial v_{0}}, \quad \forall i, a=0, \ldots, N .
$$

Proof. Let us use the same notation as in the proof of Lemma 2.2. Using that $W^{j}$ is independent of the jet variables, then the vanishing of the variational derivatives, i.e. the sum of (7) and (8) simplifies to

$$
g\left(\left(\left(\partial_{v_{a}} W^{j}\right) \Omega_{\ell}-\left(\partial_{v_{\ell}} W^{j}\right) \Omega_{a}\right) \Phi_{i}, \Phi_{j}\right)=0
$$

where we used that $\partial_{v_{a}} \Omega_{\ell}=\partial_{v_{\ell}} \Omega_{a}$, which follows from the flatness of the quantum connection. Put $\ell=0$, so that $\Omega_{\ell}=1$. Then the above identity becomes equivalent to the identity that we wanted to prove.

Proof of part b) of Theorem 1.1. According to Lemma 2.2 the Hamiltonian $H=f(v)$ is independent of the jet variables. Let us denote by $F(v)$ the gradient of $f$ with respect to the pairing $G$, i.e.,

$$
G\left(F(v), \Phi_{a}\right)=\frac{\partial f}{\partial v_{a}}
$$

A straightforward computation, similar to the proof of formula (5), yields

$$
\frac{\partial F}{\partial v_{\ell}}=\Omega_{\ell} \frac{\partial F}{\partial v_{0}}, \quad \forall \ell=0, \ldots, N
$$


and

$$
X_{H}(v)=\partial F+\frac{1}{2} \partial v \bullet F .
$$

Note that the derivation $X_{H}$ is polynomial in $v_{0}$ if and only if the gradient $F(v)$ depends polynomially in $v_{0}$. Arguing by induction on the degree of $F$ as a polynomial in $v_{0}$ we prove that

$$
F(v)=-\operatorname{Res}_{q=\infty} S(v, q) C(q) \frac{d q}{q-1}
$$

for some $C(q) \in K(X)[q]$. The above identity, formula (11), and the quantum differential equations $(q-1) \partial S(v, q)=\partial v \bullet S(v, q)$ imply the formula stated in the Theorem.

If the degree of $F$ is 0 , then recalling the differential equation (10) we get $\partial_{v_{\ell}} F=0$ for all $\ell$, so $F$ is independent of $v$. We just have to use that

$$
1=-\operatorname{Res}_{q=\infty} S(v, q) \frac{d q}{q-1} .
$$

For the induction step, note that $\partial_{v_{0}} F$ is the gradient of $-f+\partial_{v_{0}} f$. According to Lemma 2.3. the latter Poisson commutes with all $H_{i}(v, q)$, so using the inductive assumption we have

$$
\partial_{v_{0}} F(v)=-\operatorname{Res}_{q=\infty} S(v, q) C(q) \frac{d q}{q-1}
$$

for some $C(q) \in K(X)[q]$. Put

$$
\widetilde{F}(v):=-\operatorname{Res}_{q=\infty} \partial S(v, q) C(q)(q-1) \frac{d q}{q-1} .
$$

Recalling the differential equations (10) we get $\partial_{v_{\ell}} F=\partial_{v_{\ell}} \widetilde{F}$ for all $\ell$, hence $F-\widetilde{F}$ is independent of $v$. Since both $\widetilde{F}$ and $F-\widetilde{F}$ can be presented in the form (12), the gradient $F$ also has such a presentation.

\section{Construction of the topological solution}

3.1. From descendants to ancestors. The genus- 0 total ancestor potential $\overline{\mathcal{F}}_{\tau}$, $\tau \in K(X)$, is defined by

$$
\left.\overline{\mathcal{F}}_{\tau}(\mathbf{t})=\sum_{\ell, n=0}^{\infty} \sum_{d} \frac{Q^{d}}{n ! \ell !}\left\langle\mathbf{t}\left(\bar{L}_{1}\right), \ldots, \mathbf{t}\left(\bar{L}_{n}\right), \tau, \ldots, \tau\right)\right\rangle_{0, n+\ell, d},
$$

where $\bar{L}_{i}(1 \leq i \leq n)$ is the pullback of the tautological cotangent line bundle $L_{i}$ on $\overline{\mathcal{M}}_{0, n}$ via the forgetfull morphism $X_{0, n+\ell, d} \rightarrow \overline{\mathcal{M}}_{0, n}$. By definition the potential $\overline{\mathcal{F}}_{\tau}(\mathbf{t})$ is a formal power series in $\mathbf{t}, \tau$, and $Q$. Let us recall the following formula relating the ancestor and descendant potentials (see [11, Appendix B)

$$
\mathcal{F}(\mathbf{t})=\frac{1}{2}\langle\mathbf{q}(L), \mathbf{q}(L)\rangle_{0,2}(\tau)+\overline{\mathcal{F}}_{\tau}\left([S(\tau, q) \mathbf{q}(q)]_{+}\right) .
$$


where $\mathbf{q}(q):=\mathbf{t}(q)+1-q$ and []$_{+}$denotes the operation induced from the projection $K(X)(q) \rightarrow K(X)\left[q, q^{-1}\right]$.

3.2. Genus-0 reconstruction in terms of the $S$-matrix. Let us choose a formal power series $\tau:=\tau(Q, \mathbf{t})$ with coefficients in $K(X)$, such that

$$
\left.[S(\tau, q) \mathbf{q}(q)]_{+}\right|_{q=1}=0 .
$$

Since $[S(\tau, q)(1-q)]_{+}=1-q-\tau$ the above equation is equivalent to

$$
\left.[S(\tau, q) \mathbf{t}(q)]_{+}\right|_{q=1}=\tau .
$$

This is a fixed point problem for $\tau$ and we can construct a formal solution using the standard iteration procedure, e.g., put $\tau^{(0)}(Q, \mathbf{t})=0$ and set

$$
\tau^{(n+1)}:=\left.\left[S\left(\tau^{(n)}, q\right) \mathbf{t}(q)\right]_{+}\right|_{q=1}
$$

It is easy to check that as $n \rightarrow \infty$, the sequence of formal series $\tau^{(n)}(Q, \mathbf{t})$ has a limit which provides a solution to our fixed-point problem.

Lemma 3.1. Let $\tau=\tau(Q, \mathbf{t})$ be a solution to the fixed-point equation (14). Then the following formulas hold

$$
\begin{aligned}
\mathcal{F}(\mathbf{t}) & =\frac{1}{2}\langle\mathbf{q}(L), \mathbf{q}(L)\rangle_{0,2}(\tau), \\
\partial_{n, i} \mathcal{F}(\mathbf{t}) & =\left\langle\Phi_{i}(L-1)^{n}, \mathbf{q}(L)\right\rangle_{0,2}(\tau), \\
\partial_{n, i} \partial_{m, j} \mathcal{F}(\mathbf{t}) & =\left\langle\Phi_{i}(L-1)^{n}, \Phi_{j}(L-1)^{m}\right\rangle_{0,2}(\tau) .
\end{aligned}
$$

Proof. The first formula follows immediately from (13), because if $\tau$ is a solution to (14), then $\overline{\mathcal{F}}_{\tau}\left([S(\tau, q) \mathbf{q}(q)]_{+}\right)=0$. Indeed, put $\bar{q}=[S(\tau, q) \mathbf{q}(q)]_{+}$and note that the ancestor correlator

$$
\left\langle\overline{\mathbf{q}}\left(\bar{L}_{1}\right), \ldots, \overline{\mathbf{q}}\left(\bar{L}_{n}\right)\right\rangle_{0, n}(\tau)=\sum_{\ell=0}^{\infty} \sum_{d} \frac{Q^{d}}{\ell !}\left\langle\overline{\mathbf{q}}\left(\bar{L}_{1}\right), \ldots, \overline{\mathbf{q}}\left(\bar{L}_{n}\right), \tau, \ldots, \tau\right\rangle_{0, n+\ell}
$$

contains a factor of $\left(\bar{L}_{1}-1\right) \cdots\left(\bar{L}_{n}-1\right)$, which is a pullback of $\left(L_{1}-1\right) \cdots\left(L_{n}-1\right)=$ $0 \in K\left(\overline{\mathcal{M}}_{0, n}\right)$.

To prove the second identity, let us differentiate formula (13) in $q_{n, i}$ before we specialize $\tau$ to $\tau(Q, \mathbf{t})$. We get

$$
\begin{aligned}
\partial_{n, i} \mathcal{F} \quad & =\left\langle\Phi_{i}(L-1)^{n}, \mathbf{q}(L)\right\rangle_{0,2}(\tau)+ \\
& +\sum_{n=0}^{\infty} \frac{1}{n !}\left\langle\left[S(\tau, q) \Phi_{i}(L-1)^{n}\right]_{+}, \overline{\mathbf{q}}\left(\bar{L}_{2}\right), \ldots, \overline{\mathbf{q}}\left(\bar{L}_{n+1}\right)\right\rangle_{0, n+1}(\tau) .
\end{aligned}
$$

Let us specialize $\tau$ to $\tau(Q, \mathbf{t})$, then $\overline{\mathbf{q}}(1)=0$ and since $\left(L_{2}-1\right) \cdots\left(L_{n+1}-1\right)=0$ in $K\left(\overline{\mathcal{M}}_{0, n+1}\right)$ we get that the sum on the second line of the above formula vanishes. Note that the same argument can be used to prove the third formula. 
3.3. Proof of Theorem 1.3. We claim that any solution $\tau=\tau(Q, \mathbf{t})$ to the fixed point problem (14) satisfies (3) and

$$
J(\tau, 0)=1+\sum_{j=0}^{N} \partial_{0, j} \partial_{0,0} \mathcal{F}(\mathbf{t}) \Phi^{j} .
$$

The above equation follows immediately from Lemma 3.1 and the string equation

$$
\begin{aligned}
& \langle 1, \mathbf{t}(L), \ldots, \mathbf{t}(L)\rangle_{0, n+1, d}= \\
& \langle\mathbf{t}(L), \ldots, \mathbf{t}(L)\rangle_{0, n, d}+\sum_{i=1}^{n}\langle\mathbf{t}(L), \ldots, \Delta \mathbf{t}(L), \ldots \mathbf{t}(L)\rangle_{0, n, d}
\end{aligned}
$$

where the insertion $\Delta \mathbf{t}(L):=\frac{\mathbf{t}(L)-\mathbf{t}(1)}{L-1}$ is at the i-th place and the equality holds for all $d \neq 0$ or $n \geq 3$ (see [9, 11]). We just have to prove that $\tau=\tau(Q, \mathbf{t})$ is a solution to (3).

We have

$$
\partial_{n, i} J(\tau, 0)=\partial_{0,0} \sum_{j=0}^{N}\left\langle\Phi_{j}, \Phi_{i}(L-1)^{n}\right\rangle_{0,2}(\tau) \Phi^{j} .
$$

Note that if $f \in \mathbb{C}\left[q, q^{-1}\right]$, then

$$
\operatorname{Res}_{q=0, \infty} \frac{f(q) d q}{L-q}=f(L) .
$$

Therefore

$$
\left\langle\Phi_{j}, \Phi_{i}(L-1)^{n}\right\rangle_{0,2}(\tau)=\operatorname{Res}_{q=0, \infty} d q(q-1)^{n}\left\langle\Phi_{j}, \frac{\Phi_{i}}{L-q}\right\rangle_{0,2}(\tau) .
$$

By the definition of the operator series $S(\tau, q)$, we get

$$
\partial_{0,0}\left\langle\Phi_{j}, \frac{\Phi_{i}}{L-q}\right\rangle_{0,2}(\tau)=-q^{-1} \partial_{0,0} G\left(\Phi_{j}, S(\tau, q) \Phi_{i}\right) .
$$

Recall that

$$
G(a, b)=g\left(a, S(\tau, 0)^{-1} b\right)
$$

hence

$$
\partial_{0,0}\left\langle\Phi_{j}, \frac{\Phi_{i}}{L-q}\right\rangle_{0,2}(\tau)=-q^{-1} \partial_{0,0} g\left(\Phi_{j}, S(\tau, 0)^{-1} S(\tau, q) \Phi_{i}\right)
$$

and the equation (15) takes the form

$$
\partial_{n, i} J(\tau, 0)=-\partial_{0,0} \operatorname{Res}_{q=0, \infty} \frac{d q}{q}(q-1)^{n} S(\tau, 0)^{-1} S(\tau, q) \Phi_{i} .
$$

Using that $S(\tau, q)$ is a fundamental solution for $\nabla$, we get

$$
\partial_{n, i} J(\tau, 0)=\partial_{n, i} S(\tau, 0)^{-1} 1=S(\tau, 0)^{-1} \partial_{n, i} \tau
$$


and

$$
\partial_{0,0}\left(S(\tau, 0)^{-1} S(\tau, q)\right)=q S(\tau, 0)^{-1} \partial_{0,0} S(\tau, q) .
$$

The equation (17) takes the form

$$
\partial_{n, i} \tau=-\operatorname{Res}_{q=0, \infty} d q(q-1)^{n} \partial_{0,0}\left(S(\tau, q) \Phi_{i}\right) .
$$

Since $S(\tau, q)$ does not have a pole at $q=0$, the above formula reduces to the formula that we have to prove.

3.4. The Topological recursion relations. We would like to derive the Ktheoretic Topological Recursion Relations (TRR) from the fact that the solution $\tau=\tau(Q, \mathbf{t})$ to the fixed point problem (14) is a solution to the system (3) . Put

$$
\left\langle E_{1} L_{1}^{k_{1}}, \ldots, E_{r} L_{r}^{k_{r}}\right\rangle_{0, r}(\mathbf{t}):=\sum_{\ell=0}^{\infty} \sum_{d} \frac{Q^{d}}{\ell !}\left\langle\Phi_{i_{1}} E_{1} L_{1}^{k_{1}}, \ldots, E_{r} L_{r}^{k_{r}}, \mathbf{t}, \ldots, \mathbf{t}\right\rangle_{0, r+\ell, d},
$$

where $\mathbf{t} \in K(X)\left[q, q^{-1}\right]$ and the $\mathbf{t}$-insertions in the correlator should be understood as $\mathbf{t}\left(L_{r+i}\right), 1 \leq i \leq \ell$. The TRR can be stated as follows.

Proposition 3.2. If $k>0$ and $E_{2}, E_{3} \in K(X)$, then the following identity holds

$$
\begin{aligned}
& \left\langle\Phi_{i}\left(L_{1}-1\right)^{k}, E_{2} L_{2}^{k_{2}}, E_{3} L_{3}^{k_{3}}\right\rangle_{0,3}(\mathbf{t})= \\
& \sum_{a, b=0}^{N}\left\langle\Phi_{i} L_{1}\left(L_{1}-1\right)^{k-1}, \Phi_{a}\right\rangle_{0,2}(\mathbf{t}) G^{a b}(\mathbf{t})\left\langle\Phi_{b}, E_{2} L_{2}^{k_{2}}, E_{3} L_{3}^{k_{3}}\right\rangle_{0,3}(\mathbf{t}),
\end{aligned}
$$

where $G^{a b}(\mathbf{t})$ are the entries of the matrix inverse to the matrix $G(\mathbf{t})=\left(G_{a b}(\mathbf{t})\right)_{a, b=0}^{N}$ defined by

$$
G_{a b}(\mathbf{t}):=g_{a b}+\left\langle\Phi_{a}, \Phi_{b}\right\rangle_{0,2}(\mathbf{t}) .
$$

Proof. According to Lemma 3.1 we have

$$
\left\langle E_{2} L_{1}^{k_{2}}, E_{3} L_{2}^{k_{3}}\right\rangle_{0,2}(\mathbf{t})=\left\langle E_{2} L_{1}^{k_{2}}, E_{3} L_{2}^{k_{3}}\right\rangle_{0,2}(\tau) .
$$

Let us write $\mathbf{t}(q)=\sum t_{k, i} \Phi_{i}(L-1)^{k}$. Differentiating the above identity by $t_{k, i}$ we get

$$
\left\langle\Phi_{i}\left(L_{1}-1\right)^{k}, E_{2} L_{2}^{k_{2}}, E_{3} L_{3}^{k_{3}}\right\rangle_{0,3}(\mathbf{t})=\left\langle\partial_{k, i} \tau, E_{2} L_{2}^{k_{2}}, E_{3} L_{3}^{k_{3}}\right\rangle_{0,3}(\tau) .
$$

Recalling that $\tau$ is a solution to (3) we get

$$
\partial_{k, i} \tau=-\operatorname{Res}_{q=0, \infty} d q(q-1)^{k-1} \partial_{0,0} \tau \bullet S(\tau, q) \Phi_{i} .
$$

For $k=0$, since $S(\tau, q)$ does not have a pole at $q=0$ or $\infty$, the above equation gives

$$
\partial_{0, i} \tau=\partial_{0,0} \tau \bullet \Phi_{i} .
$$


Therefore, we have

$$
\partial_{k, i} \tau=-\operatorname{Res}_{q=0, \infty} d q(q-1)^{k-1} \sum_{a, b=0}^{N} G\left(S(\tau, q) \Phi_{i}, \Phi_{a}\right) G^{a b}(\tau) \partial_{0, b} \tau .
$$

Recalling the definition of the $S$-matrix we get

$$
-\operatorname{Res}_{q=0, \infty} d q(q-1)^{k-1} G\left(S(\tau, q) \Phi_{i}, \Phi_{a}\right)=\operatorname{Res}_{q=0, \infty} d q q(q-1)^{k-1}\left\langle\Phi_{a}, \frac{\Phi_{i}}{L-q}\right\rangle_{0,2}(\tau) .
$$

The above residue is precisely $\left\langle\Phi_{a}, \Phi_{i} L(L-1)^{k-1}\right\rangle_{0,2}(\tau)$ (see formula (16)). The RHS of (18) turns into

$$
\sum_{a, b=0}^{N}\left\langle\Phi_{i} L_{1}\left(L_{1}-1\right)^{k-1}, \Phi_{a}\right\rangle_{0,2}(\tau) G^{a b}(\tau)\left\langle\partial_{0, b} \tau, E_{2} L_{2}^{k_{2}}, E_{3} L_{3}^{k_{3}}\right\rangle_{0,3}(\tau) .
$$

Note that

$$
\left\langle\partial_{0, b} \tau, E_{2} L_{2}^{k_{2}}, E_{3} L_{3}^{k_{3}}\right\rangle_{0,3}(\tau)=\partial_{0, b}\left\langle E_{2} L_{1}^{k_{2}}, E_{3} L_{2}^{k_{3}}\right\rangle_{0,2}(\tau),
$$

so to complete the proof it remains only to recall the 2-jet identity in Lemma 3.1 .

3.5. Geometric proof of TRR. The TRR can be proved geometrically (see 11, Appendix A) using the standard cotangent lines comparison techniques. Namely, let $\pi: X_{0, \ell+3, d} \rightarrow \overline{\mathcal{M}}_{0,3}$ be the map that forgets the map to the target $X$ and the last $\ell$ marked points. Then we have

$$
L_{1}=\bar{L}_{1} \otimes \mathcal{O}(D), \quad \bar{L}_{1}=\pi^{*} L_{1},
$$

where $D \subset X_{0, n+3, d}$ is the divisor consisting of stable maps for which the irreducible component that caries the first marked point is contructed by $\pi$. Since $\mathcal{O}(-D)=1-\mathcal{O}_{D}$ and $\bar{L}_{1}=1$ we get

$$
L_{1}-1=L_{1} \otimes \mathcal{O}_{D}
$$

The divisor $D$ is a union of divisors $D_{i}$ obtained from an appropriate gluing map

$$
X_{0, \ell_{i}^{\prime}+2, d_{i}^{\prime}} \times_{\Delta} X_{0, \ell_{i}^{\prime \prime}+3, d_{i}^{\prime \prime}} \rightarrow X_{0, \ell+3, d}
$$

where $\Delta \subset X \times X$ is the diagonal and the fiber product on the LHS is defined via the evaluation maps corresponding to the marked points to be glued. The structure sheaf $\mathcal{O}_{D}$ can be expressed in terms of the structure sheaves $\mathcal{O}_{D_{i}}$ using the inclusion-exclusion principle (see [4])

$$
\mathcal{O}_{D}=\sum_{i} \mathcal{O}_{D_{i}}-\sum_{i, j} \mathcal{O}_{D_{i} \cap D_{j}}+\sum_{i, j, k} \mathcal{O}_{D_{i} \cap D_{j} \cap D_{k}}-\cdots
$$


Writing the first insertion of the correlator on the LHS of TRR as $\Phi_{i}\left(L_{1}-\right.$ $1)^{k-1} L_{1} \otimes \mathcal{O}_{D}$ and using the above formula for $\mathcal{O}_{D}$ we get precisely the RHS of TRR, where $G^{a b}(\mathbf{t})$ comes as the $(a, b)$-entry of

$$
g^{-1}+\sum_{\ell=1}^{\infty}(-1)^{\ell} g^{-1}\left(G(\mathbf{t}) g^{-1}\right)^{\ell} .
$$

Using TRR and Lemma 3.1 we can give an alternative proof of Theorem 1.3 . The idea is to use TRR to simplify the RHS of formula (15), which would allow us to express the derivatives $\partial_{n, i} \tau(Q, \mathbf{t})$ in terms of the $S$-matrix. The argument is straightforward, so we skip the details.

\section{REFERENCES}

[1] B. Dubrovin. Geometry of 2D topological field theories. In: "Integrable systems and quantum groups" (Montecatini Terme, 1993), 120-348, Lecture Notes in Math., 1620, Springer, Berlin, 1996.

[2] B. Dubrovin and S. Novikov. The Hamiltonian formalism of one-dimensional systems of hydrodynamic type and the Bogolyubov-Whitham averaging method. Soviet Math. Dokl., vol. 270.4 (1983): 665-669.

[3] B. Dubrovin and Y. Zhang. Normal forms of hierarchies of integrable PDEs, Frobenius manifolds and Gromov - Witten invariants. arXiv:math/0108160.

[4] A. Givental. On the WDVV equation in quantum K-theory. Mich. Math. J., vol. 48 (2000): 295-304.

[5] A. Givental and V. Tonita. Hirzebruch-Riemann-Roch theorem in true genus-0 quantum K-theory. In Symplectic, Poisson, and Noncommutative Geometry, Cambridge Univ. Press, Math. Sci. Res. Inst. Publications, vol. 62, (2014): 43-92.

[6] Yu. Manin. Frobenius Manifolds, Quantum Cohomology, and Moduli Spaces. AMS Colloquium Publications, vol. 47, Providence, RI, 1999, 303pp.

[7] C. Hertling and Yu. Manin. Weak Frobenius manifolds. Int. Math. Res. Notices, vol. 6 (1999): 277-286

[8] H. Iritani, T. Milanov, and V. Tonita. Reconstruction and convergence in Quantum K-theory via difference equations. IMRN, vol. 11 (2015): 2887-2937.

[9] Y.-P. Lee. Quantum K-theory I. Foundations. Duke. Math. J., vol. 121, no. 3 (2004): 389-424.

[10] Y.-P. Lee. A formula for Euler characteristics of tautological line bundles on the DeligneMumford moduli spaces. IMRN, vol. 8 (1997): 393-400.

[11] V. Tonita and H.-H. Tseng. Quantum orbifold Hirzerbruch-Riemann-Roch theorem in genus zero. arXiv: 1307.0262 .

Kavli IPMU (WPI), UTIAS, The University of Tokyo, Kashiwa, Chiba 277-8583, JAPAN

E-mail address: todor.milanov@ipmu.jp

Humboldt Univerität, Rudower Chaussee 25 Raum 1425, Berlin 10099, Germany

E-mail address: valentin.tonita@hu-berlin.de 